\section{International Scientific Journal Theoretical \& Applied Science}

p-ISSN: 2308-4944 (print) e-ISSN: 2409-0085 (online)

Year: $2016 \quad$ Issue: 6 Volume: 38

Published: $30.06 .2016 \quad$ http://T-Science.org
Moldir Ermekbaevna Aliyeva Doctoral student, Kazakh National Pedagogical University after Abay, Almaty

Victor Vladimirovich Kazachonak Professor, Doctor of Pedagogical Sciences, Belarusian State University, Minsk

Kanat Mukashevich Mukashev Professor, Doctor of Physical and Mathematical Sciences, Kazakh National University after al - Farabi, Almaty

kanat-kms@mail.ru

\title{
SOME QUESTIONS OF INTERACTION OF ELECTROMAGNETIC WAVES WITH THE ELECTRONIC SUBSYSTEM OF THE ATOM
}

Abstract: In an article for an explanation of the photoelectric effect phenomenon offers new ways of its theoretical analysis in the course of physics. Considerable attention is paid to the analysis of wave processes arising from the interaction of photons with electrons substances. It described the process of absorption of a photon by an electron. The possibilities of absorption of a photon by a stationary free electron and photon absorption of a photon moving electrons. It is shown that an electron at rest can not absorb a photon. Analyzed many aspects of the presentation of the phenomenon of the photoelectric effect.

Key words: electromagnetic waves, photons, atoms, electrons, interaction, photoelectric effect.

Language: English

Citation: Aliyeva ME, Kazachonak VV, Mukashev KM (2016) SOME QUESTIONS OF INTERACTION OF ELECTROMAGNETIC WAVES WITH THE ELECTRONIC SUBSYSTEM OF THE ATOM. ISJ Theoretical \& Applied Science, 06 (38): 131-133.

Soi: http://s-o-i.org/1.1/TAS-06-38-27 Doi: crossef http://dx.doi.org/10.15863/TAS.2016.06.38.27

Photoelectric effect (photo effect) belongs to the special processes of nature that widely used in engineering. At the same time it is one of the most important problems of Physics during studying the nature of the light proving its wave and quantum characteristics. Despite this fact, in the course of Physics this phenomenon is stated as a simple liberation of the electrons from the surface when light beam gets on the metal, and at the best case, completed with the Stoletov's known laws [1].

In the textbooks for the enhanced studying of Physics [2,3] the condition of the liberation of the electron from a metal is determined by the A.Einstein equation:

$$
\begin{gathered}
\varepsilon_{v}=h v=A_{0}+\frac{m \vartheta^{2}}{2} ; \\
\left(\varepsilon_{v}=h v>A_{0}\right),
\end{gathered}
$$

where, $A_{o}-$ work of the liberation of the electron from the metal that is phenomenon of the photo effect can only occur if the photon energy will be more than the work of the liberation of the electron. But we are not talking about what the state of the electrons can absorb the photons to overcome the potential barrier of the surface.

Thus, in practice, when studying the phenomenon of the photoelectric remains as unknown effect for the pupil - a quantum of light (photon) is absorbed by a free electron or an electron associated. It seems that even teachers have a vague notion. It is evident therefore that for the elucidation of this question there is a method to shed light on the physical side of this problem. In this regard, in order to describe clearly the process of the absorption of the photon by the electron we consider that their interaction takes place as the clash. From the point of view of the corpuscular wave-particle duality, such an approach is not considered as erroneous. Then the process takes place according to the laws of the conservation of the energy and momentum. Applying these laws we analyze the question of the absorption of a photon electron according to three aspects.

1. Possibility of the absorption of a photon by $a$ free electron of the photon.

As a result of the collision with the stationary photon the free electron make a move. Let its speed be $\vartheta_{1}$ (figure 1). 


\begin{tabular}{l|lrl|l|ll} 
& ISRA (India) & $=\mathbf{1 . 3 4 4}$ & SIS (USA) & $=\mathbf{0 . 9 1 2}$ & ICV (Poland) & $=\mathbf{6 . 6 3 0}$ \\
Impact Factor: & ISI (Dubai, UAE) $=\mathbf{0 . 8 2 9}$ & PVHIL (Russia) $=\mathbf{0 . 2 3 4}$ & PIF (India) & $=\mathbf{1 . 9 4 0}$ \\
& GIF (Australia) & $\mathbf{0 . 5 6 4}$ & ESJI (KZ) & $=\mathbf{1 . 0 4 2}$ & IBI (India) & $\mathbf{4 . 2 6 0}$
\end{tabular}

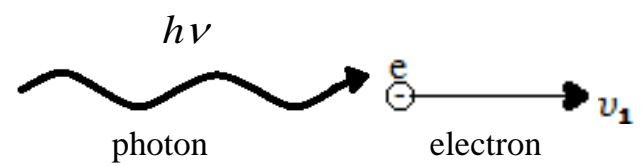

Figure 1 - The impact of a photon on a stationary electron.

In accordance to the law of the conservation of energy this movement can be described as follows:

$$
h v+m_{0} c^{2}=m c^{2}
$$

$h v$ - is the energy of the photon; $m_{o}$ - is the rest mass of the electron; $m$ - is the electron mass in a motion.

Expressing body weight in a motion through a change in the rest mass, the equation (1) is transformed into:

$$
h v+m_{0} c^{2}=\frac{m_{0} c^{2}}{\sqrt{1-\left(\vartheta_{1} / c\right)^{2}}}
$$

Solving the last equation find the speed of the electron after the absorption of a photon:

$$
\vartheta_{1}=\frac{c \sqrt{v h\left(h v+2 m_{0} c^{2}\right)}}{h v+m_{0} c^{2}}
$$

The same value of the speed of the electron can be determined based on the law of the conservation of momentum. Let the speed of the electron in this case be $\vartheta^{\prime}$ :

$$
\frac{h v}{c}=\frac{m_{0} \vartheta^{\prime}}{\sqrt{1-\left(\vartheta^{\prime} / c\right)^{2}}}
$$

In the left part of this equation $h v / c$ - is the initial quantity of a motion (momentum) of the photon, on the right one is a momentum of the electron after the absorbing of the photon. From this equation it is determined the speed of the moving electron:

$$
\vartheta^{\prime}=\frac{h v c}{\sqrt{(h v)^{2}+\left(m_{0} c^{2}\right)^{2}}} .
$$

If the resting electron in a collision absorbs a photon then the values of the speeds determined using the laws of conservation of energy and momentum must be equal to each other. However, comparing the right sides of the expressions (3) and (4) it is not difficult to verify that $\vartheta_{1} \neq \vartheta^{\prime}$. Therefore we can conclude that a motionless electron cannot absorb the photon.

The possibility of the absorption of a photon by a moving electron.

Of course, in the general case the direction of the movement of the moving electron and the photon before the collision can be different. But to facilitate the resolution of this question we will focus on the case when the directions of the mutual relative motion of the electron and the photon will be perpendicular (figure 2):

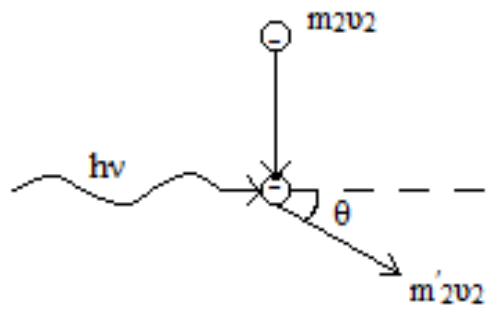

Figure 2 - The collision of a photon with a moving electron.

Let the speed of the electron before the collision be $\vartheta_{2}$, weight of $m_{2}=\frac{m_{0}}{\sqrt{1-\left(\vartheta_{2} / c\right)^{2}}}$.

As a result of a collision the electron absorbs a photon and changes its direction of a motion. The electron moves forward at angle $\angle \theta$ to the direction of a motion of the photon. As a result, its speed will be $\vartheta$ and the weight of $m_{2}^{\prime}=\frac{m_{0} c^{2}}{\sqrt{1-(\vartheta / c)^{2}}}$. The speed of the electron after the collision is determined on the basis of the law of the conservation of the energy from the relation:

$$
h v+m_{2} c^{2}=\frac{m_{0} c^{2}}{\sqrt{1-(\vartheta / c)^{2}}}
$$




\begin{tabular}{|c|c|c|c|c|c|c|}
\hline Impact Factor: & $\begin{array}{l}\text { ISRA (India) } \\
\text { ISI (Dubai, UAF } \\
\text { GIF (Australia) } \\
\text { JIF }\end{array}$ & $\begin{array}{l}=1.344 \\
=0.829 \\
=0.564 \\
=1.500\end{array}$ & $\begin{array}{l}\text { SIS (USA) } \\
\text { PИНЦ (Russia) } \\
\text { ESJI (KZ) } \\
\text { SJIF (Morocco) }\end{array}$ & $\begin{array}{l}=0.912 \\
=0.234 \\
=1.042 \\
=2.031\end{array}$ & $\begin{array}{l}\text { ICV (Poland) } \\
\text { PIF (India) } \\
\text { IBI (India) }\end{array}$ & $\begin{array}{l}=6.630 \\
=1.940 \\
=4.260\end{array}$ \\
\hline
\end{tabular}

Is seen to be:

$$
\vartheta=\frac{c \sqrt{v h\left(h v+2 m_{0} c^{2}\right)+\left(m_{2}^{2}-m_{0}^{2}\right) c^{4}}}{h v+m_{2} c^{2}}
$$

Now to calculate the same quantities on the basis of the law of the conservation of a momentum the following equation is compiled that determines the horizontal component of a momentum:

$$
\frac{h v}{c}=\frac{m_{0}}{\left.\sqrt{1-\left(\vartheta^{\prime} / c\right.}\right)^{2}} \vartheta^{\prime} \cos \theta
$$

Here $\vartheta^{\prime}$ - is the speed defined by this equation. Component of a momentum in the vertical direction

$$
m_{2} \vartheta_{2}=\frac{m_{0}}{\sqrt{1-\left(\vartheta^{\prime} / c\right)^{2}}} \vartheta^{\prime} \sin \theta
$$

Squaring both sides of equations (7) and (8) and summing them term by term find the speed of an electron $\vartheta^{\prime}$ after the absorption of a photon:

$$
\vartheta^{\prime}=\frac{c \sqrt{(v h)^{2}+\left(m_{2} \vartheta_{2} c\right)^{2}}}{(h v)^{2}+\left(m_{0} c^{2}\right)^{2}+\left(m_{2} \vartheta_{2} c\right)^{2}} .
$$

And in this case equating the right sides of the equations (6) and (9) make sure that $\vartheta_{1} \neq \vartheta^{\prime}$. Since in this case the collision process of a photon with a moving free electron does not obey to the laws of the conservation of the energy and a momentum one may come to the conclusion that the moving free electrons cannot absorb the photons.

The photons created by the nuclear reactions and decay of nuclei are called gamma quantum. Despite of the fact that their energy (0.1 to $100 \mathrm{MeV})$ is much greater than the photon energy of the light beam, they don't lose their wave properties. When interacting with the matter those photons are involved in the three processes as the photoelectric effect, coherent scattering and the production of the electron - positron pairs [4]. In these conditions the low energy quanta leads to the phenomenon of the photoelectric effect and the photon spends all its energy on the interaction with the bound electron. Only in the case where obtained electron energy will be greater than its binding energy with the atom the electron leaves the atom and the phenomenon of the photoelectric effect will occur. Denoting the excess energy as $\mathrm{W}$, we will express the law of the conservation of the energy the corresponding to this case by the following equation:

$$
h v+m_{2} c^{2}-W=\frac{m_{0} c^{2}}{\sqrt{1-(\vartheta / c)^{2}}} .
$$

Is seen to be

$$
h v=\left[\frac{m_{0} c^{2}}{\sqrt{1-(\vartheta / c)^{2}}}-m_{0} c^{2}\right]+W
$$

Given that $\vartheta<<c$ this expression transforms:

$$
h=m_{0} c^{2}\left[1+\frac{1}{2}(\vartheta / c)^{2}\right]-m_{0} c^{2}+W,
$$

or

$$
h v=\frac{1}{2} m_{0} \vartheta^{2}+W
$$

It is easy to verify that the last expression is the Einstein equation describing the condition of the phenomenon of the photoelectric effect. From this point of view it is possible to analyze many aspects of presentation of the phenomenon of the photoelectric effect and thereby to quicken interest of the pupils to Physics and Physical phenomena.

\section{References:}

1. Myakishev GY, Bukhovtsev BB (2002) Physics, class 11. -A.: Kazakhstan, 240 p.

2. (2002) Physics, class 11 (under the editorship of A. A. Pinsky).-M.: Prosveshcheniye, 432 p.
3. (1995) Elementary textbook of Physics, vol. III. (under the editorship of G. S. Landsberg).Moscow: CJSC "Shrike". 422 p.

4. Walter AK, Zalyubovsky II (1978) Nuclear Physics. -Kharkiv. Vyshcha shkola. 424 p. 